\title{
Consideration of reinforcement mechanism in the short fiber mixing granular materials by granular element simulations
}

\author{
Kentaro Mori ${ }^{1, \star}, K^{2}$ jeni Kaneko ${ }^{1, \star \star}$, and Yutaka Hashizume ${ }^{1, \star \star \star}$ \\ ${ }^{1}$ Hachinohe Institute of Technology, 88-1 Obiraki, Myo, Hachinohe City, Japan
}

\begin{abstract}
The short fiber mixing method is well known as one of the method to improve the strength of granular soils in geotechnical engineering. Mechanical properties of the short fiber mixing granular materials are influenced by many factors, such as the mixture ratio of the short fiber, the material of short fiber, the length, and the orientation. In particular, the mixture ratio of the short fibers is very important in mixture design. In the past study, we understood that the strength is reduced by too much short fiber mixing by a series of tri-axial compression experiments. Namely, there is "optimum mixture ratio" in the short fiber mixing granular soils. In this study, to consider the mechanism of occurrence of the optimum mixture ratio, we carried out the numerical experiments by granular element method. As the results, we can understand that the strength decrease when too much grain-fiber contact points exist, because a friction coefficient is smaller than the grain-grain contact points.
\end{abstract}

\section{Introduction}

There is short fiber mixing reinforced soil which improves material strength by mixing short fibers with granular soil material whose performance is insufficient. In this method, short fibers made of polyethylene, polyester or natural palm having a length of about several $\mathrm{cm}$ are used. By mixing short fibers with granular soils, strength and toughness is improved. It is a basic concept of improvement that the short fiber mixing reinforced soil adds macroscopic cohesion by resisting tensile force of fibers. Many studies are carried out by a macroscopic viewpoint [1]. However, since various causes such as length, diameter, rigidity, mixture ratio and orientation of short fibers are complicatedly involved in the short fiber mixing reinforced soil, it is very difficult to elucidate its mechanical characteristics and reinforcement mechanism only by macroscopic viewpoint. There are several studies using DEM based on a microscopic viewpoint [2]. However, especially, relationship between the mixture ratio and the mechanical properties is not identified clearly.

In the theme of relationship between the mixture ratio and the mechanical properties of short fiber mixing reinforced soil, our research group carried out a series of laboratory tri-axial compression experiments using two kinds of silica sands and polyethylene short fibers [3]. As the results of these experiments, we can say that the macroscopic strength of short fiber mixing granular soils decreases when the mixture ratio is increased too much. In other words, the maximum strength exists for the short

\footnotetext{
^e-mail: g139044@hi-tech.ac.jp

$\star \star$ e-mail: kaneko@hi-tech.ac.jp

$\star \star \star$ e-mail: hashizume@hi-tech.ac.jp
}

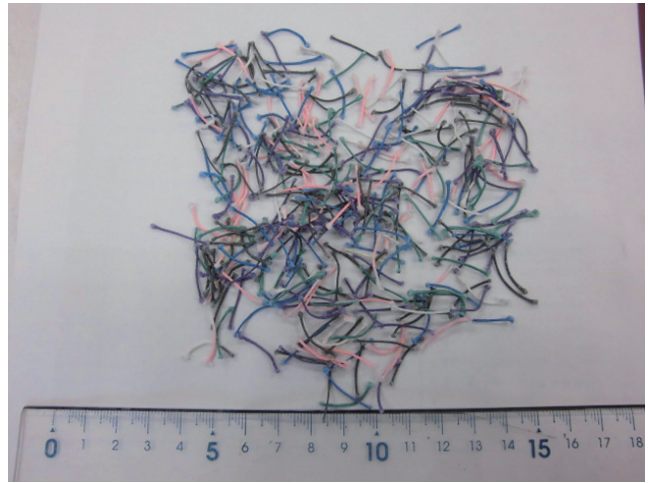

Figure 1. Short fibers used in our experiments

fiber mixture ratio and "the optimum mixture ratio" of the short fibers exists.

In this study, by microscopic numerical experiments, we examine the mechanism that the optimum mixture ratio exists. In our numerical experiments, we employ the Granular Element Method (GEM) proposed by Kishino [4, 5], as one of the discrete element method. Although GEM is similar to Distinct Element Method (DEM), GEM solves the quasi-static equilibrium equation of discrete system.

\section{Outline of results of tri-axial compression tests}

Focusing on the relationship between the mixture ratio of the short fiber and shear strength in the short fiber mixing reinforced soil, we carried out a series of the tri-axial compression test. Figure 1 shows short fibers, which are fish- 


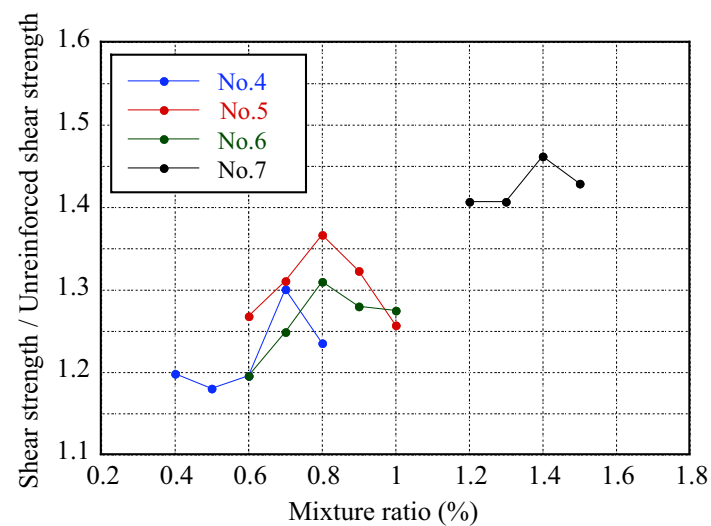

Figure 2. Shear strength and mixture ratio

ing line made of polyethylene, used in our experiments as the reinforcement material. The diameter of the short fiber is about $0.52 \mathrm{~mm}$ and length is about $20 \mathrm{~mm}$. We used four silica sands called No.4, No.5, No.6, and No.7. The average grain size of silica sands is different while the shape of grain size distribution is very similar. The mixture ratio of fiber used in the reinforced specimens is defined as the ratio of the weight of fibers $\left(W_{F}\right)$ and the dry weight of sand $\left(W_{S}\right)$ :

$$
w_{f}=\frac{W_{F}}{W_{S}} \times 100(\%)
$$

The test specimen was made by sufficiently compacting so that the density becomes $90 \%$ of maximum dry density of sand. The confining pressure is $200 \mathrm{kN} / \mathrm{m}^{2}$ in all of the experimental cases.

Figure 2 shows the relationship between maximum principal stress difference and mixture ratio of short fibers obtained from the results in each case of tri-axial compression tests. In Fig. 2, normalized maximum principal stress difference of the vertical axis normalizes maximum principal stress difference in each case in that of the sands only. In all cases, shear strength defined by maximum principal stress difference is increased by mixing short fibers. However, shear strength decreases when exceeding a specific mixture ratio, and the reinforcement effect by mixing short fibers tends to become small. Consequently, it became clear that optimum mixture ratio related to shear strength in the short fiber mixing granular soils exists. Figure 3 shows the relationship between optimum mixture ratio of the short fiber and average grain size. As the sand has smaller particle size, necessary quantity of the fiber to get the maximum reinforcement effect increases.

From the results of the tri-axial compression tests, it is considered that when short fibers are mixed in granular media, two effects are mainly performed internally. One of them is a positive effect of restraining motion of grains by the tension of short fibers. However the granular medium without the cohesion is weak to tensile stress generally, the macroscopic cohesive strength is obtained by mixing fiber. The other one is a negative effect that the frictional force between the short fibers and the soil grains is lower than the friction between the soil particles, so that the macroscopic frictional force decreases. In the particulate ma-

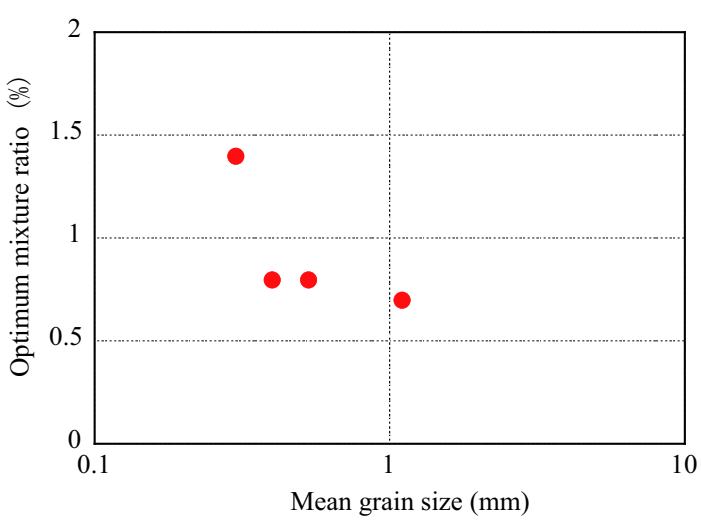

Figure 3. Optimum mixture ratio and mean grain size

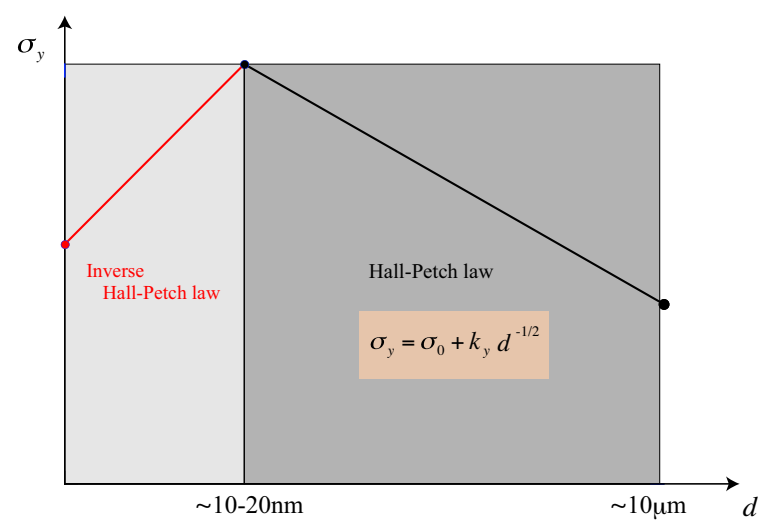

Figure 4. Outline of Hall-Petch law

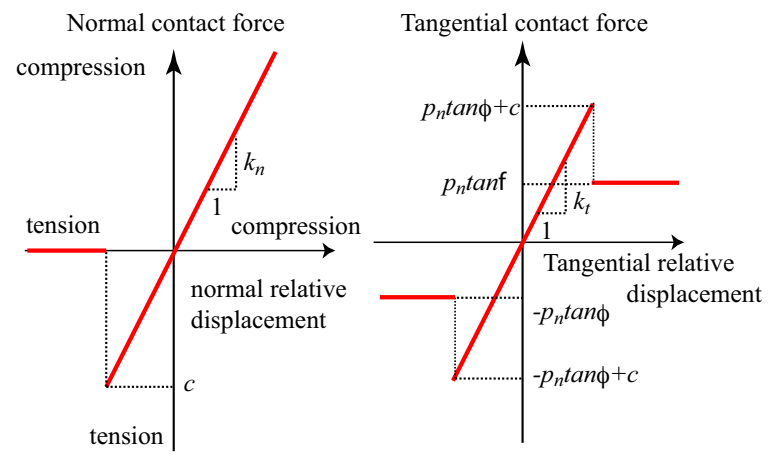

Figure 5. Fiber contact model for particle contacts

terial which mixed short fibers in surplus, we can assume that macroscopic strength may decrease because the negative effect becomes dominant than the positive effect. This assumption was considered from Hall-Petch law and inverse Hall-Petch law in the field of the metal materials.

Figure 4 shows the relationship between grain size and yield stress in Hall-Petch and inverse Hall-Petch law schematically. Generally, strength of polycrystalline metal increases when crystal particle size becomes small. This is called Hall-Petch equation, which is expressed as follows [6],

$$
\sigma_{Y}=\sigma_{0}+k_{Y} d^{-1 / 2} .
$$

Where $\sigma_{Y}$ is the yield stress, $\sigma_{0}$ and $k_{Y}$ are the material constants, and $d$ is the crystal grain size. Namely the yield 


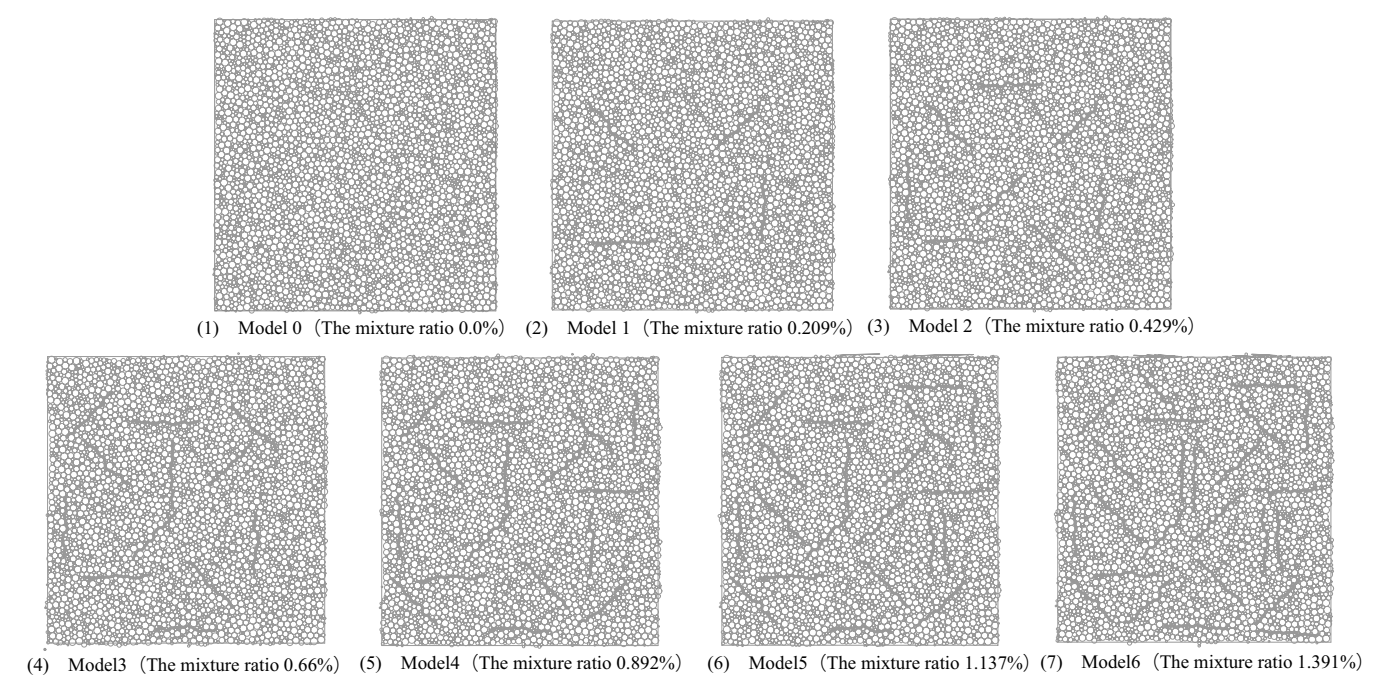

Figure 6. Analysis models

stress of a polycrystalline metal is in proportion to $d^{-1 / 2}$. The strength of the each crystal grain becomes strong as the size of the crystal grain becomes small, because the dislocation motion is more obstructed. However when the grain size is too small, the yield stress tends to decrease conversely. Because the strength of grain boundary, which is weaker than that of crystal grain, becomes more dominant for macroscopic strength, the macroscopic strength decreases when a crystal grain becomes small too much. This is called inverse Hall-Petch law. From such a law, it may be assumed that similar phenomena may also occur in short fiber mixing reinforced soil.

\section{Numerical experiments by GEM}

In this section, to explain the hypothesis in section 2, we carry out a series of numerical experiments by using 2-dimensional Granular Element Method with periodic boundary conditions [5].

\subsection{Outline}

At first, we explain the outline of numerical experiments of the short fiber mixing reinforced granular materials. This is aiming to explore the relationship between the shear strength and the mixture ratio.

As a model, the fiber connecting the small particles, is to be used to provide the tensile resistance between them. The schematic diagram of the contact model between the particles is shown in Figure 5. In the conventional GEM for analyzing the aggregate of only dry grains, if the normal relative displacement between the particles was tensile, the contact force between the particles would be determined as the non-contact. In the case of using fiber, the contact state would be maintained until the tensile normal contact force arrives at the tensile strength ( $c$ in Figure 5). In the tangential direction, the simple Coulomb's friction law is adopted as shown in

$$
p_{t} \leq p_{n} \tan \phi
$$

where $p_{n}$ is the normal contact force, $p_{t}$ is the tangential one and $\phi$ is the friction angle between particles. For the fiber particles-fiber particles contact, the tangential constraint model is taken as shown in

$$
p_{t} \leq p_{n} \tan \phi+c
$$

To represent the small bending stiffness of short-fibers the small tangential spring constant is used compared to the normal direction.

In general, it is considered that the friction coefficient of short fibers is smaller than that of soil particles. The friction coefficient of short fibers has a large influence on the negative effect. Therefore, in this study, we carry out the numerical experiments of two cases which changed a coefficient of friction of the short fiber. In the first case, the friction coefficient of short fibers is equal to that of soil particles. It is assumed that there is no negative effect in this case. In the second case, we use a very small friction coefficient of the fiber to assume the case which has a very large negative effect. For each parameter on contact points between the soil particle and the short fiber particle, the average value of each parameter was used. Regarding $c$ representing the tensile strength of short fibers, a huge value is used because short fibers never break during the tri-axial compression test.

Figure 6 shows numerical models prepared with different mixture ratios. In this figure, the initial state was created with an isotropic stress of $0.2 \mathrm{MPa}$. Model 0 , which has 5223 disk particles with $0.2 \mathrm{~mm}-1 \mathrm{~mm}$ diameters, is a model that does not mix short fibers. Models 1 to 6 were prepared by increasing short fibers particles and decreasing soil particles on the basis of model 0 , but the void ratio was assumed to be almost constant in each model. On the assumption of a short fiber having a diameter of $0.5 \mathrm{~mm}$ and a length of $20 \mathrm{~mm}, 41$ short fiber particles having a particle diameter of $0.52 \mathrm{~mm}$ were connected and modeled. Numerical models are analyzed by stress control with constant lateral pressure. 


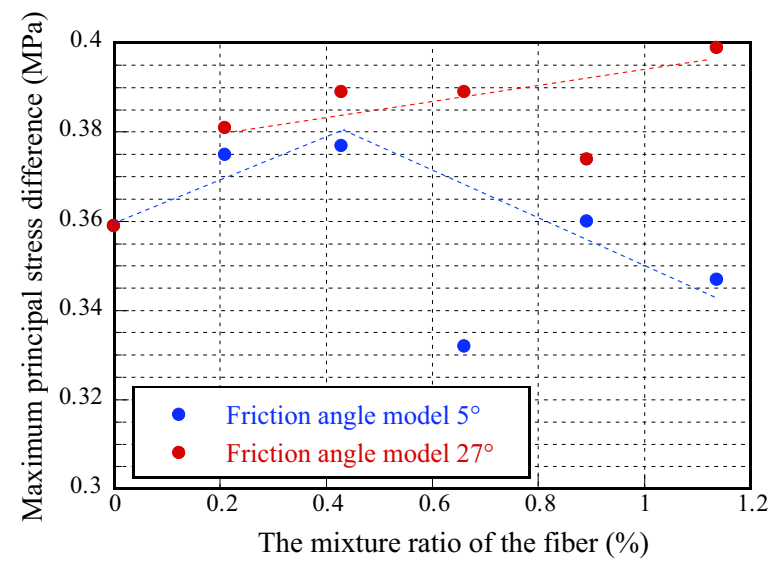

Figure 7. Mixture ratio and maximum principal stress difference

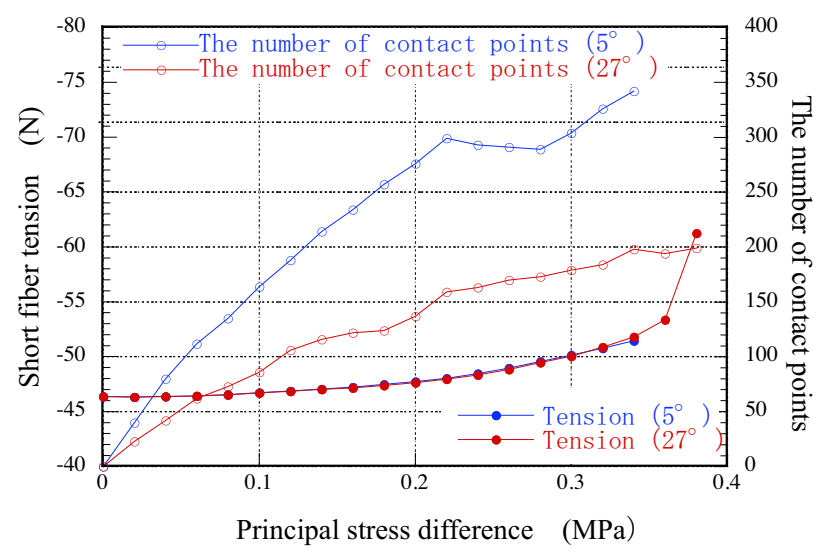

Figure 8. Fiber tension and slip occurrence (model 5)

\subsection{Results}

Figure 7 shows the relationship between the short fiber mixture ratio and the maximum principal stress difference obtained by changing the inter-particle friction angle of each model to $5^{\circ}$ and $27^{\circ}$. When the friction angle between the particles of the short fibers is set to $27^{\circ}$ which is the same as the soil particles, the maximum principal stress difference is larger at any mixture ratio compared to the case of the short fibers are not mixed. However, even if mixed rates increase, the maximum principal stress difference changes little. On the limited conditions, it seems that when the friction angle is $5^{\circ}$, even when the result of model 3 is regarded as an error, the maximum principal stress difference decreases with an increase in the mixture ratio of short fibers. It is considered that the shear strength of short fiber mixing reinforced soil is determined mainly by the balance between the positive effect due to the tension of the short fibers and the negative effect due to the decrease in the coefficient of friction between the soil particles. When the coefficient of friction between the short fiber particles is made small, it is considered that this is an increase in the contact point of a small coefficient of friction due to an increase in short fibers.

Figure 8 shows the relationship between the change in short fiber tension accompanying shearing loading in model 5 (the mixture ratio is $1.137 \%$ ) and the change in the number of contact points where slip between short fibers and soil particles occurs. This is a model which has the largest influence difference of friction angle between particles. As can be seen from this figure, the tension is not so different even if the friction angle between the short fiber particles is changed, and almost the same tension is generated. On the other hand, as for the number of contact points between the short fibers and the soil particles where the slip is occurring, it can be seen that there are very many contact points where slipping occurs from the initial stage of loading. From the above, it is considered that the optimum mixture ratio with respect to the shear strength of the particulate material mixed with the short fibers appears because the negative action exceeds the positive action.

\section{Conclusions}

We clarified the appearance mechanism of optimum mixture ratio in reinforced soil by mixing short fiber from the results of the tri-axial compression tests and the numerical experiments. It may be possible that the optimum mixture ratio appears because the negative effect by mixing short fibers in surplus exceeds than the positive effect. In future, we plan to demonstrate a clear trend by conducting more numerical experiments. It is very important that there is an optimal solution for the mixture ratio of short fiber mixing reinforced soil. And it can contribute to the elucidation of the improvement mechanism of the mechanical properties of the granular material by mixing of short fibers.

\section{Acknowledgements}

This work was supported by JSPS KAKENHI Grant Number JP16K06493.

\section{References}

[1] A. Diambra, et al., Experiments and modeling, Geotextiles and Geomembranes, 28, pp. 238-250 (2010).

[2] E. Ibraim \& K. Maeda, New Horizons in Earth Reinforcement, Otani et al. eds., pp. 387-393 (2008).

[3] S. Nozoe, et al.,Powders \& Grains 2013, pp. 305-308 (2013).

[4] Kishino, Y.,Powders and Grains, pp. 323-330, (1989).

[5] Kaneko, K., et al. Int. J. Solids Struct., 40, pp.40434069, (2003).

[6] W. D. Callister, Jr.John Wiley \& Sons, Inc, (2007). 